\title{
A Short Term Load Forecasting Algorithm Based on Gray Elman Neural Network and Genetic Algorithm
}

\author{
Baoyi Wang ${ }^{1, a}$, Zheng Wang ${ }^{1, b}$, Shaomin Zhang ${ }^{1, c}$ \\ ${ }^{1}$ School of Control and Computer Engineering, North China Electric Power University, Baoding \\ 071003, Hebei Province, China \\ aemail: wangbaoyiqj@126.com, bemail:774588763@qq.com, 'email: zhangshaomin@126.com
}

Keywords: gray theory; genetic algorithm; Elman neural network; load forecasting

\begin{abstract}
Short term power load sample is highly variable, and the influence factors are not determined, the data sample is little. In view of the characteristics of the load, we combine the Grey Theory and Elman neural network to predict the short-term power load. Because the gray neural network convergence is slow, We introduce the genetic algorithm to the gray Elman neural network optimization, and propose the genetic algorithm to optimize the gray Elman neural network algorithm, the genetic algorithm to optimize the gray Elman neural network algorithm is applied to short-term load forecast. Experimental results show that the prediction accuracy is improved. The algorithm achieves fast convergence, and it is feasible and effective.
\end{abstract}

\section{Introduction}

There are many factors that affect short-term power system load, such as weather factors, the regional economic structure, seasonal factors and other random factors, including meteorological factors on the load is very important. Now, with the meteorological department technology continues to improve and perfect, they can provide real-time weather information relevant to the electricity sector, which is a big help to improve the power system load precision work.

The literature [7] compares the BP algorithm and ELMAN algorithm by wavelet denoising before and after. The literature [9] uses fuzzy gray relational clustering method to select similar days load, uses wavelet neural network to forecast load. The literature [10] proposed a BP neural network fuzzy clustering technology and artificial neural network model combining. The literature [11] proposes short-term load forecasting based on improved ultimate learning machine, through data comparison, MELM to be more accurate than the ELM and OS-ELM algorithm.

Since the 1980s, as a new discipline gray system theory are maturing, its distinguishing feature is used to study less data, methods of uncertainty. Theoretically it is more suitable for load forecasting that has a non-linear changes and influenced by many factors. In the gray modeling the most representative is $\mathrm{GM}(1,1)$ model, which is gray dynamic model for time series. The time series data directly into differential equations, using the system information so abstract models to quantify, and thus in the absence of knowledge of the properties of the system output forecasting system, but the prediction deviation is large, can not be determined by uncertainty prediction . In contrast, the artificial neural network learning is powerful, which Elman neural network can approximate any function, with a strong ability to fit. It can learn to mutation data. This article intends to put the gray theory and Elman neural network to build predictive algorithms, and improved by genetic algorithm. The algorithm is applied to the power grid load forecasting, prediction of power load a certain area, and the results to be compared.

\section{Grey GM $(1,1)$ model}

In the gray theory, all systems can be gray sequence generated by some weakening of its randomness, show its regularity. Grey power load forecasting is the application of gray system theory, through the establishment of the original data processing and gray model, we discover and master the law of development of the load. On the future status of the load quantitatively predict 
essentially the "process immediately" as a "gray process", "then the variable" as a "gray variable."

Column with the original data $X(0)=(X(0)(1), X(0)(2), \ldots, X(0)(n)), \mathrm{n}$ is the number of data. GM $(1,1)$ model is established based on $X(0)$, to achieve the prediction function, the steps are as follows:

(1) the accumulation of raw data in order to weaken the volatility and randomness of random sequence to obtain new data series:

$$
X^{(1)}=\left\{X^{(1)}(1), X^{(1)}(2), \ldots, X^{(1)}(n)\right\}
$$

Wherein, $X(1)(t)$ represents the accumulation of all the data before the data of several, that is $X$ (1) is the 1 -AGO sequence of $X(0)$.

$$
x^{(1)}(t)=\sum_{k=1}^{t} x^{(0)}(k) \square t=1,2, \ldots, n
$$

(2) According to the $X(1)(t)$ to establish a first-order linear differential equation, that is GM $(1,1)$ model.

$$
\frac{d x^{(1)}}{d t}+a x^{(1)}=u
$$

Wherein, $a, u$ is undetermined coefficient, called the development coefficient and gray action quantity, $a$ valid range is (2,2), and note the matrix consisting of $a$ and $u$ as gray parameter. Just obtaining parameters $a$ and $u$, we can find $X(1)(t)$, and then find the next predicted value of $X(0)$.

(3) The gray parameter into Formula 3, and Formula 3 is solved as follows:

$$
\hat{X}^{(1)}(t+1)=\left(\hat{X}^{(0)}(1)-\frac{u}{a}\right) e^{-a t}+\frac{u}{a}
$$

Since gray parameter is determined by the method of least squares approximation, so the $\hat{X}^{(1)}(t+1)$ is an approximate expression, in order to distinguish the original sequence, it is referred to as $\hat{X}^{(1)}(t+1)$. Where $t$ is the time series.

\section{Elman neural network model}

Currently in the field of short-term load forecasting BP neural network is the most widely used, but the BP neural network is actually a static feedforward networks. In the neural network, the modeling of dynamic time becomes static modeling problems, which inevitably leads to many problems.It is able to reflect the system dynamic characteristics more directly and vividly that using dynamic neural network in the load forecasting system directly. Elman recurrent network is a typical dynamic neural networks, compared to the BP neural network, Elman neural network in addition to the input layer,, hidden layer and output layer, there is a receiving layer. Receiving layer is mainly used for feedback coupling between the inner layer or layers, making it possible to express the delay between the input and output over time, which is equivalent to a delay operator, and therefore need a dynamic equations to describe, and feedforward networks only to realize nonlinear mapping. Because of this feedback, the network has a memory function.The structure of Elman neural network is shown in Figure 1.

Due to undertake layer, feed-forward connecting portion can be connected right to amend, and a recursive part is fixed, that can not be learning correction. From block diagram, the structural unit output at time $k$, is equal to a times of the implied layer output value of the moment $k-1$, that is:

$$
x_{c, l}(k)=a \llbracket x_{c, l}(k-1)+x_{l}(k-1), l=1,2, \ldots, n
$$

Wherein, $x_{c, l}(k)$ and $x_{l}(k)$ denote the output of the i-th hidden layer unit, a is self-connecting feedback gain factor. When a fixed zero, the network is a standard Elman network, when a not equal to zero, the network is the modified Elman network. Mathematical model of the network is:

$$
\begin{aligned}
& x(k)=f\left(w_{1} x_{c}(k)+w_{2} u(k-1)\right) \\
& x_{c}(k)=a \square x_{c}(k-1)+x(k-1) \\
& y_{k}=g\left(w_{3} x(k)\right)
\end{aligned}
$$


Wherein, $\mathrm{f}(\mathrm{x})$ to take on more of sigmoid function, that is

$f(x)=\frac{1}{1+e^{-x}}$

$\mathrm{g}(\mathrm{x})$ to take on more of a linear function, that is:

$y_{k}=w_{3} x(k)$

Elman neural network deviation function is defined as:

$\mathrm{E}=\frac{1}{2}\left(y_{d}(k)-y(k)\right)^{T}\left(y_{d}(k)-y(k)\right)$

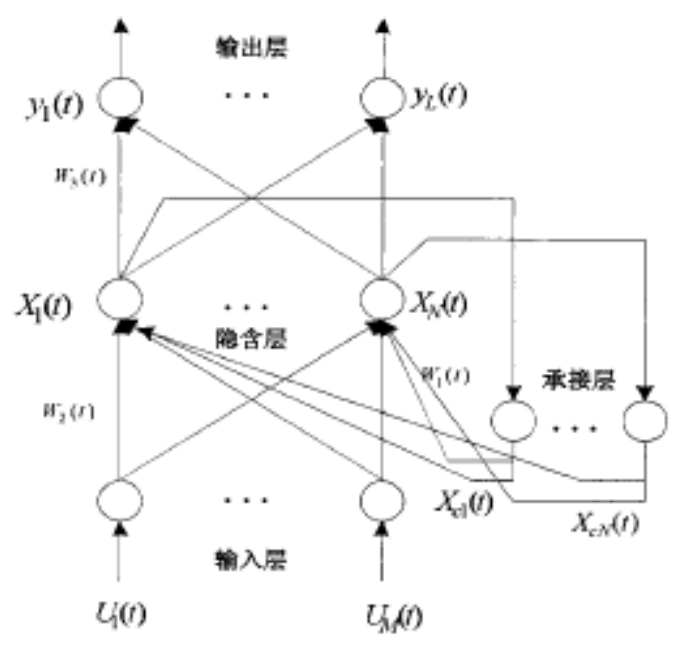

Figure 1 Elman neural network structure

\section{Genetic gray Elman neural network load forecasting algorithm}

Since the weights and thresholds of the gray Elman neural network random initialization, it is easy to fall into local optimum, and genetic algorithm has global convergence, the use of genetic algorithm to optimize the initial weights and thresholds gray Elman neural network, both to overcome the neural network architecture selected uncertainty, but also to improve the performance of network convergence and convergence rate.

As can be seen from the above formulas, weights and thresholds gray Elman neural network model have direct or indirect relationship with the parameters a and $\mathrm{u}$, as long as the initial values of the parameters are given, you can initialize all weights and thresholds. Genetic algorithm optimization factors include population initialization, fitness function, select Options, crossover, and mutation. In this paper, the individual coding using real-coded; take individual correspondence Grey neural network forecasting deviation for the individual fitness function; use roulette as the selection operation. Genetic Algorithms gray Elman neural network algorithm flow is shown in Figure 2.

\section{Genetic gray Elman neural network load forecasting process}

(1) Input and output variable selection

Input variables of the algorithm uses a total of 10, including the day before the predicted point maximum load, two days before the predicted point maximum load, maximum load on the same day the previous week, the maximum load before the day the previous week, the same prediction point, the same prediction from the previous week one day after the maximum point load, the maximum load on the same day the previous month, the maximum load forecast a month ago in the same spot the day before, in January after the maximum load point before the same day forecast predicted date type, predicted average daily temperature; output variable is the day of maximum load forecast. 
(2) Data normalization process

(3) The gray Elman neural network training data and genetic algorithm optimization.

Gray Elman neural network uses back-propagation method to train data, the training steps are as follows:

Initialize the network based on the training data, initialization gray parameters and calculate the initial weights of the network.

For each input sequence, calculate each output,select the excitation function, then calculate the prediction deviation of the network between the output and the desired output. Adjusting the weights and thresholds based on deviation.

Determine whether the train arrival times, is the end, or else return to step 2.

(4) Deviation Analysis

Forecast results using percentage deviation (percentage error, PE) and the mean absolute deviation (mean absolute percent error, MAPE) to test output, wherein $X_{r}$ represents the actual load value, $X_{f}$ represents the estimated load value. PE and MAPE expressions are as follows:

$$
\begin{aligned}
& P_{E}=\frac{X_{f}-X_{r}}{X_{r}} \times 100 \\
& M_{A P E}=\frac{1}{31} \sum_{i=1}^{31} \frac{\left|X_{r}-X_{f}\right|}{X_{r}}
\end{aligned}
$$

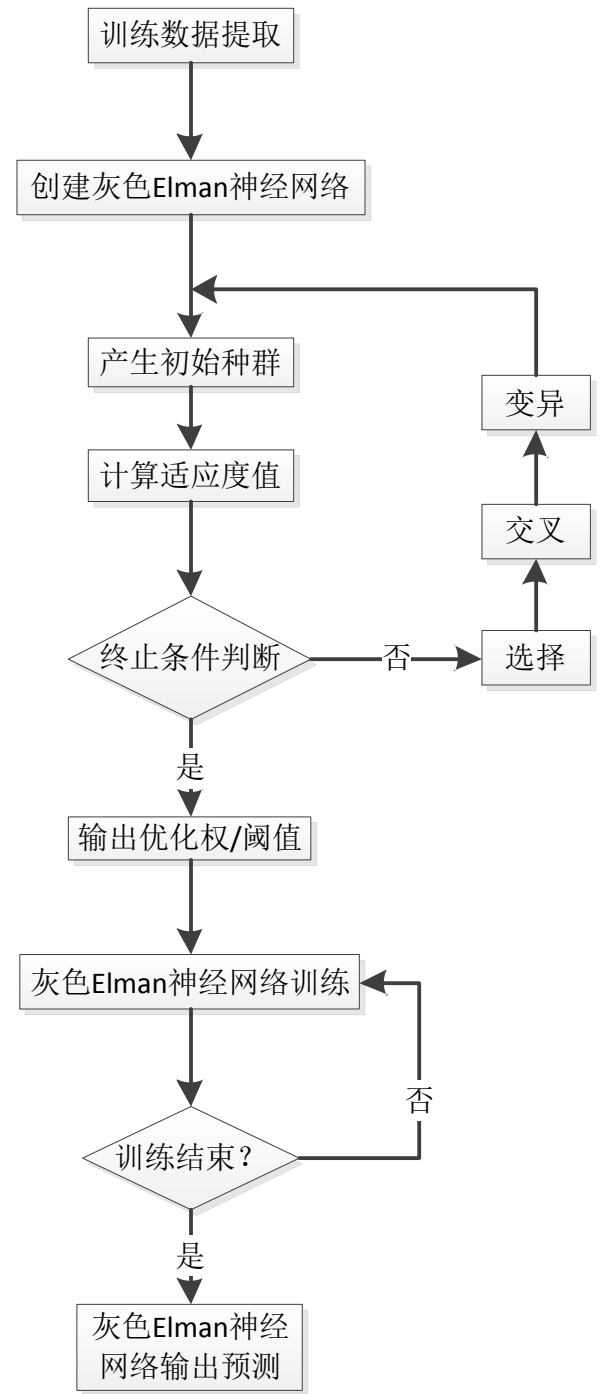

Figure 2 Gray Elman neural network genetic algorithm optimization flowchart 


\section{Result Analysis}

With the above method, select an area 1997, 1998 real load data. Data provided by the European Smart Technology Network (EUNITE). We predict the maximum daily load 31 days in January 1999.

Table 1 lists the initial value, the initial value obtained through genetic algorithm optimization gray Elman neural network, take the population size 30, 150 iterations. Table 2 shows predicted results. Figure 3 and Figure 4 shows the results of load forecasting and load results percentage deviation.

From the above examples can be drawn, when utilizing Elman neural network and gray Elman neural network load forecasting, the deviation is large, its MAPE were 3.56\% and 3.16\%, while the use of genetic gray Elman conduct load forecasting, forecast accuracy has been very large increase, which was $1.44 \%$ MAPE.

Table 1 best initial parameter

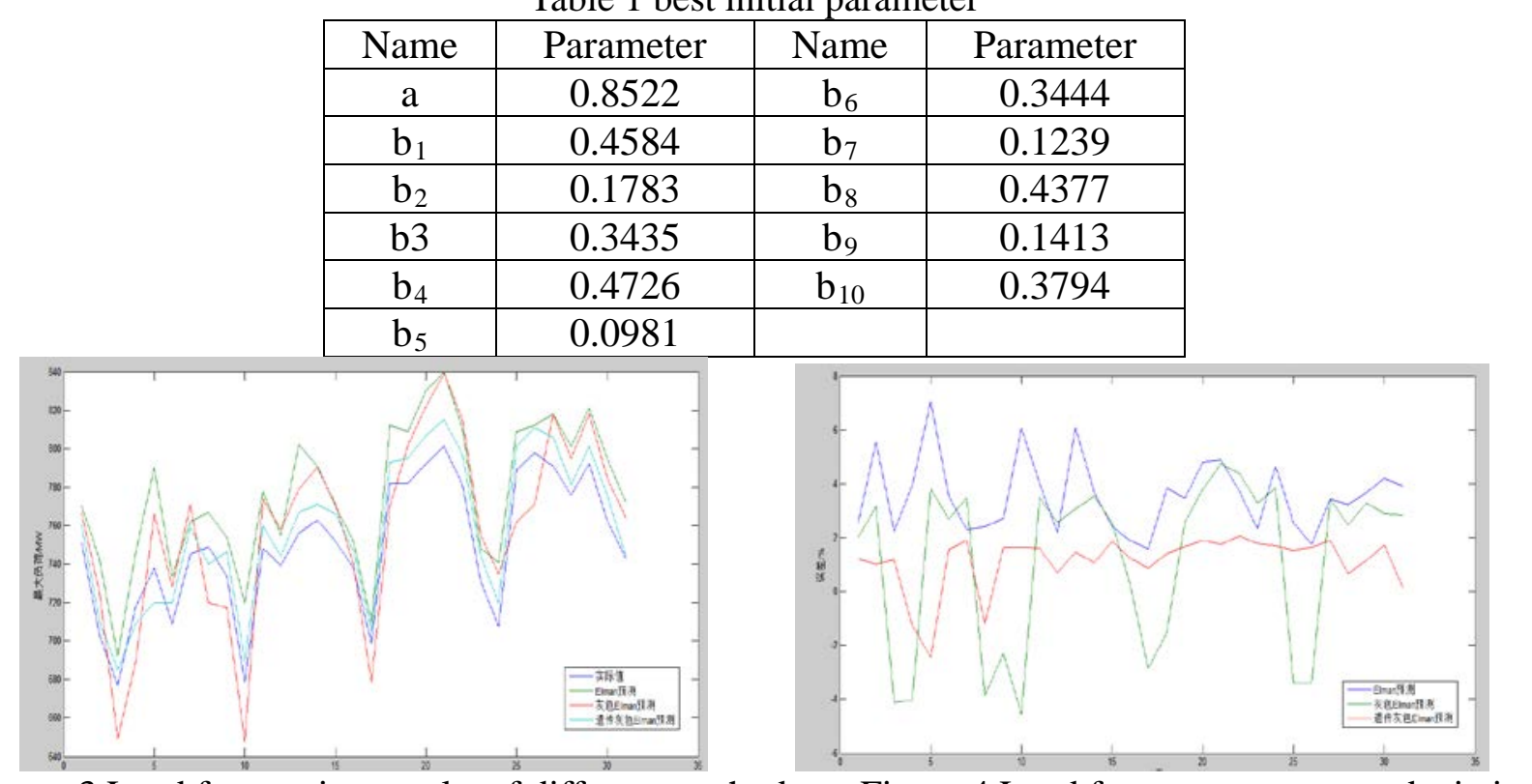

Figure 3 Load forecasting results of different methods

Figure 4 Load forecast percentage deviation

\section{Conclusion}

In the smart grid, a variety of factors influence the accuracy of load forecasting is not the same, it is difficult to establish the mathematical model to describe. In this paper, considering the impact of the date and weather factors, gray Elman neural network model can weaken the randomness of the data, the neural network is highly nonlinear. We use these characteristics to predict the short-term load. Genetic algorithms optimize the network. Improve the accuracy of predictions. Examples of results demonstrate the effectiveness of the proposed method.

\section{Acknowledgement}

In this paper, the research was sponsored by the Scientific Research Project of Hebei Province (Project No. Z2012077).

\section{References}

[1] Liao Nihuan. Summary of forecasting methods of power system short-term load [J]. Power system protection and control system, 2011, Volume 39 (1): 147-152.

[2] Cai Xia, Xing Jun. Summary of Power System Load Forecasting Method [J]. Information Research, 2010, Volume 36(6): 5-7. 
[3] Liu Xiaojuan. Forecasting model and its application in power system based on intelligent method of load [D]. Donghua University, 2013.

[4] Cai Jianbiao. Cloud Computing Platform for Smart Grid Load Forecasting Based [D]. Hunan University, 2013.

[5] Wang Dapeng, Grey forecasting long term load forecasting model and applied research [D]. Wuhan: Huazhong University of Science and Technology, 2013.

[6] Meng Mengmeng, Prediction of short-term heating network load RBF neural network [D]. Harbin: Harbin Institute of Technology, 2013.

[7] Jiang Jinan. Short Term Load Forecasting Decomposition and Artificial Neural Networks [D]. Guangxi University, 2013.

[8] Zeng Ming,Lv Chunquan etc. Based on bacterial communities chemotaxis optimization LSSVM term load forecasting method [J]. China Electrical Engineering, 2011,31(34): 93-99.

[9] Shi Biao, Li Yuxia, Yu Xinhua. Short Term Load Forecasting Fuzzy Neural Network - based on improved particle swarm [J]. Systems Engineering Theory and Practice, 2010,30(1): 157-166.

[10] Li Zuo, Zhou Buxiang, Lin Nan. Curve classification and short-term load forecasting based on fuzzy clustering algorithm with Japan improved BP load characteristics [J]. Power system protection and control system, 2012,40(3): 56-60.

[11] Mao Li, Wang Yuntao, Liu Xingyang. Short-term load forecasting method based on improved Extreme Learning Machine [J]. Power system protection and control system, 2012,40(20): 140-144.

[12] Zhang Daiyuan. Based on neural network algorithm distributed parallel computing [J]. Systems Engineering and Electronics, 2010, 32(2): 386-391.

[13] Zhang Ping, Pan Xueping, Xue Wenchao. Based on the short-term load wavelet fuzzy gray clustering and BP neural network prediction[J]. Electric Power Automation Equipment, 2012,32(11): 121-125.

[14] Wang Baoyi, Zhao Shuo, Zhang Shaomin. Distributed power load forecasting algorithm cloud computing and Extreme Learning Machine [J]. Power System Technology, 2014,38(2): 526-531.

[15] Xiao Bai, Zhou Chao,Mu Gang. Spatial load forecasting method Summary and Outlook [J]. China Electrical Engineering, 2013,33(25): 78-92.

[16]Liu Rong. Elman neural network based on short-term load forecasting [D]. Zhejiang University, 2013.

[17] Zhao Yun. Based on Distributed Learning Neural Network Intrusion Detection Algorithm [D]. Harbin Polytechnic University, 2014.

[18] Sifeng Liu, Xie Naiming. Grey System Theory and Applications (6th Edition) [M]. Science Press,2013. 